OUTP-99-41P

hep-th/9909018

\title{
Infinitely Coloured Black Holes
}

\author{
Nick E. Mavromatos* and Elizabeth Winstanley \\ University of Oxford, Department of Physics, Theoretical Physics, 1 Keble Road, \\ Oxford, OX1 3NP, United Kingdom.
}

\begin{abstract}
We formulate the field equations for $S U(\infty)$ Einstein-Yang-Mills theory, and find spherically symmetric black-hole solutions. This model may be motivated by string theory considerations, given the enormous gauge symmetries which characterize string theory. The solutions simplify considerably in the presence of a negative cosmological constant, particularly for the limiting cases of a very large cosmological constant or very small gauge field. The situation of an arbitrarily small gauge field is relevant for holography and we comment on the AdS/CFT conjecture in this light. The black holes possess infinite amounts of gauge field hair, and we speculate on possible consequences of this for quantum decoherence, which, however, we do not tackle here.
\end{abstract}

September 1999

* Currently also at Theory Division, CERN, CH-1211 Geneva 23, Switzerland. 


\section{Introduction}

The discovery of the Bartnik-McKinnon self-gravitating particle-like structure [1] has opened the way for the construction of several hairy black hole structures in Einstein theories coupled to non-Abelian gauge fields [2]. In this work we would like to address some issues concerning black hole solutions in $S U(\infty)$ gauge theories. Our interest in such structures will be motivated by string theory considerations. String theories are known to be characterized by infinite-dimensional gauge symmetries, relating the various string levels. Such infinite symmetries have been argued [3, 4] to be sufficient for the maintainance of quantum coherence during black hole (quantum) evaporation. The basic idea behind such conjectures is that infinite symmetries are sufficient to encode all the information inside a stringy black hole, since the latter can be in any one of an infinity of excited massive-string level states during the evaporation, thereby accounting for the enormous number of states that appears to characterize the Bekenstein-Hawking entropy formula for macroscopic black holes. This obviously could not have been achieved in a local field theory with a finite number of conserved charges.

However, the point of view of ref. [4] was that simply infinite-dimensional symmetries were not by themselves sufficient to maintain coherence, during the complex process of evaporation/decay in string theory. Studies in prototypes of two-target-space-dimensional string theories have revealed that the latter possess black hole structures which are characterized [4, 5] by specific infinite-dimensional symmetries, namely $W_{\infty}$ symmetries [0], in their target space. Such symmetries have the peculiar property of preserving a twodimensional phase-space manifold under target-time translations, which in the particular case of two-dimensional strings may be identified with the phase space of scalar massless matter in the two-dimensional black hole space time. Hence, during black-hole quantum evaporation, which according to ref. [4], is a non-thermal phenomenon associated with the decay of excited black hole (internal) string states, quantum coherence of the string matter is maintained as a result of geometrical properties of the black hole hair itself (W-hair).

Although this phenomenon seems to be a novel way of tackling the coherence issue, however, the fact that in ref. [1] has been explicitly demonstrated in two-dimensional black holes has the drawback that it might be connected strictly to the two-dimensional space time. Such objections can be answered, of course, by making an analogy of the two-

dimensional stringy black hole with an effective theory arising in realistic four-dimensional theories of quantum gravity upon restricting oneself to spherically-symmetric space-time configurations; but then the challenge remains to construct explicitly string theories in four dimensions with this property. 
Motivated by these considerations, we would like in this note to take a different viewpoint, and consider black holes in standard Einstein four-dimensional space-times, which however capture some of the essential features of the above-mentioned two-dimensional stringy black holes, namely the infinite-dimensional character of the gauge hair, as well as its area-preserving nature, which was argued to be responsible for two-dimensional quantum coherence [1]. At this point we should stress that the $W$-hair of ref. [4] was quantum hair [6], arguably measured by Aharonov-Bohm type experiments, and therefore pertaining to phases in the respective matter wave functions, whereas in this paper we are concerned with purely classical hair. Nevertheless, some of the features we find, specifically the holographic properties, may be related to the issue of quantum coherence in the sense of ref. [1].

Gauge theories with gauge group $S U(N), N \rightarrow \infty$, remarkably share these features, since gauge transformations in the large- $N$ limit yield an ancestor of the $W_{\infty}$ algebra, according to the analysis of ref. [7]. Indeed, in this limit the non-Abelian commutators of such gauge transformations become classical-like Poisson brackets, which have the property of preserving the area of an "internal" sphere, $S^{2}$, pertaining to the gauge group variables. For details on this construction we refer the reader to the literature [7]; here we shall make use only of the results relevant for our purposes, namely the technicalities involved in taking the limit $N \rightarrow \infty$. Black hole solutions of the Einstein-Yang-Mills (EYM) equations for finite $N$ have been discussed in [8]. However, as was pointed out there, the finite- $N$ analysis could not be extended smoothly to the case $N \rightarrow \infty$. It is the purpose of this article to establish the existence of $S U(\infty)$ classical gauge hair in the EYM black-hole system.

The area-preserving nature of the infinite- $N$ gauge hair is an encouraging sign for the maintainance of quantum coherence during the evaporation process; however, to prove this conjecture one should study the precise mechanism behind the evaporation, which in view of the absence of a satisfactory four-dimensional quantum theory of gravity, is not feasible at present. Nevertheless, as we shall show in this article, the analytic formulation of black hole solutions with infinite gauge hair simplifies greatly in the presence of a negative cosmological constant (no matter how small). We have arbitrarily small gauge field hair (which is not possible in the absence of a cosmological constant). This implies that anti-de Sitter geometries may be used as "regulators" for the $S U(\infty)$ EYM black holes with zero cosmological constant, and therefore one can appeal to the concept of holography in the sense of [9]. This means that the behaviour of the gauge field in the bulk (i.e. outside the event horizon) is determined by the structure at infinity (boundary). Anti-de Sitter spacetimes have recently attracted great attention in view of their relevance to the conjectured equivalence between some conformal field theories on their boundary with the classical 
bulk structure (AdS/CFT correspondence) [10, 11]. The above-mentioned holographic point of view, together with the specific area-preserving properties of the $S U(\infty)$ gauge group, makes this model an interesting classical hair analogue of the $W$-hair picture of [4]. Whether it survives at the full quantum level remains an open question.

The structure of the present paper is as follows. In section 2, we review briefly the mathematical formalism behind Einstein-Yang-Mills systems, by concentrating on the construction of the field equations for spherically symmetric black hole solutions for $S U(\infty)$ gauge group. We take carefully the limit $N \rightarrow \infty$, by following the same procedure as ref. [7], which led to the area-preserving property of the $S U(\infty)$ gauge transformations. We include a cosmological constant $\Lambda$ in our model. In section 3 we consider the limit in which the cosmological constant is large and negative, since in this limit it is known that the $S U(2)$ system simplifies significantly [12]. We perform an asymptotic expansion in $\epsilon=1 / \Lambda$ and obtain analytic solutions to first order in $\epsilon$, which reveal many of the properties we expect the black holes to possess in general. We also consider the solutions for a very small gauge field, when the field equations again simplify considerably. As already mentioned, this situation is of particular interest for our ideas concerning holography [9] and the AdS/CFT correspondence [10, 11].

\section{Ansatz and field equations for $S U(\infty)$ Gauge Field}

\subsection{Mathematical Construction of the Gauge Field}

It is the purpose of this section to study in detail the mathematical construction of an $S U(N)$ gauge field, extending in a non-trivial way the flat-space-time approach of ref. [7]. First we shall keep $N$ finite, and at the end we shall take the limit $N \rightarrow \infty$ in a similar way as the one considered in [7]. The mathematical issue of how $S U(\infty)$ is constructed is rather subtle, but we shall not focus on these difficulties. Instead, we shall assume that the construction in [7] is well-defined and take that as our definition of $S U(\infty)$. We restrict our attention to space-times which are static and spherically symmetric, and shall therefore use the analysis of the spherically symmetric $S U(N)$ gauge field [13]. Throughout this paper, the metric has signature $(-,+,+,+)$. We shall retain the gravitational coupling constant $\kappa$ but set $c=1$. 
Following [0, 14] one starts from an irreducible representation of the $s u(N)$ Lie algebra, spanned by $N \times N$ Hermitian matrices $S_{i}$ :

$$
\begin{aligned}
& S_{1}=\frac{1}{2}\left(\begin{array}{cccccc}
0 & \sqrt{N-1} & 0 & \cdots & 0 & 0 \\
\sqrt{N-1} & 0 & \sqrt{2(N-2)} & \cdots & 0 & 0 \\
0 & \sqrt{2(N-2)} & 0 & \cdots & 0 & 0 \\
\vdots & \vdots & \vdots & \vdots & \vdots & \vdots \\
0 & 0 & 0 & \cdots & 0 & \sqrt{N-1} \\
0 & 0 & 0 & \cdots & \sqrt{N-1} & 0
\end{array}\right) \\
& S_{2}=\frac{1}{2}\left(\begin{array}{cccccc}
0 & -i \sqrt{N-1} & 0 & \cdots & 0 & 0 \\
i \sqrt{N-1} & 0 & -i \sqrt{2(N-2)} & \cdots & 0 & 0 \\
0 & i \sqrt{2(N-2)} & 0 & \cdots & 0 & 0 \\
\vdots & \vdots & \vdots & \vdots & \vdots & \vdots \\
0 & 0 & 0 & \cdots & 0 & -i \sqrt{N-1} \\
0 & 0 & 0 & \cdots & i \sqrt{N-1} & 0
\end{array}\right) \\
& S_{3}=\frac{1}{2}\left(\begin{array}{ccccc}
N-1 & 0 & 0 & \ldots & 0 \\
0 & N-3 & 0 & \ldots & 0 \\
0 & 0 & N-5 & \ldots & 0 \\
\vdots & \vdots & \vdots & \vdots & \vdots \\
0 & 0 & 0 & \ldots & -N+1
\end{array}\right)
\end{aligned}
$$

which satisfy the commutation relations

$$
\left[S_{i}, S_{j}\right]=i \epsilon_{i j k} S_{k} .
$$

From (프) one can construct the 'raising' and 'lowering' matrices:

$$
\begin{aligned}
S_{+} \equiv S_{1}+i S_{2}= & \left(\begin{array}{ccccccc}
0 & \sqrt{N-1} & 0 & & 0 & \cdots & 0 \\
0 & 0 & \sqrt{2(N-2)} & 0 & \cdots & 0 \\
0 & 0 & 0 & \sqrt{3(N-3)} & \cdots & 0 \\
\vdots & \vdots & \vdots & & \vdots & \vdots & \vdots \\
0 & 0 & 0 & & 0 & \ldots & \sqrt{N-1} \\
0 & 0 & 0 & & 0 & \ldots & 0
\end{array}\right) \\
S_{-} \equiv S_{1}-i S_{2} & =\left(\begin{array}{ccccccc}
0 & 0 & 0 & \ldots & 0 & 0 \\
\sqrt{N-1} & 0 & 0 & \ldots & 0 & 0 \\
0 & \sqrt{2(N-2)} & 0 & \ldots & 0 & 0 \\
\vdots & \vdots & \vdots & \vdots & \vdots & \vdots \\
0 & 0 & 0 & \ldots & \sqrt{N-1} & 0
\end{array}\right) .
\end{aligned}
$$


The most general spherically symmetric $S U(N)$ gauge potential may be written in the differential form (where all matrices are functions of the radial Schwarzschild co-ordinate r) [13]

$$
\hat{\mathcal{A}}=A^{(N)} d t+B^{(N)} d r+\frac{1}{2}\left(C^{(N)}-C^{(N) \dagger}\right) d \theta-\frac{i}{2}\left[\left(C^{(N)}+C^{(N) \dagger}\right) \sin \theta+D^{(N)} \cos \theta\right] d \phi
$$

where $D^{(N)}=\operatorname{Diag}\left\{k_{1}, \ldots, k_{N}\right\}$, with $k_{1} \geq k_{2} \geq \ldots \geq k_{N}$ integers whose sum is zero. The $N \times N$ matrix $C$ is strictly upper triangular, with complex entries satisfying $C_{i j}^{(N)} \neq 0$ only if $k_{i}=k_{j}+2$; and $C^{\dagger}$ is its Hermitian conjugate. The anti-Hermitian matrices $A, B$ commute with $D$. In addition, $D$ must satisfy the commutation relations:

$$
[D, C]=2 C, \quad\left[D, C^{\dagger}\right]=-2 C^{\dagger} .
$$

A suitable irreducible ansatz is to take [13]:

$$
D^{(N)}=\operatorname{Diag}\{N-1, N-3, \ldots-N+3,-N+1\} .
$$

The matrices $A, B$ are now diagonal with trace zero, and their entries can be written as:

$$
A_{j j}^{(N)}=i\left[-\frac{1}{N} \sum_{k=1}^{j-1} k \mathcal{A}_{k}^{(N)}+\sum_{k=j}^{N-1}\left(1-\frac{k}{N}\right) \mathcal{A}_{k}^{(N)}\right]
$$

and similarly for $B^{(N)}$. Here $\mathcal{A}_{k}^{(N)}$ are real functions. The only non-vanishing entries for $C^{(N)}$ are:

$$
C_{j, j+1}^{(N)}=\omega_{j} e^{i \gamma_{j}}, \quad j=1, \ldots N-1
$$

for real functions $\omega_{j}, \gamma_{j}$ depending on $r$. In this case, $D=2 S_{3}$, and

$$
A^{(N)}=a_{0} I+a_{1} S_{3}+\ldots+a_{N-1} S_{3}^{N-1}
$$

with a similar expression for $B^{(N)}$. The coefficients $a_{i}(r)$ are purely imaginary. In addition,

$$
C^{(N)}=c_{0} S_{+}+c_{1}\left[S_{+} S_{3}+S_{3} S_{+}\right]+\ldots+c_{N-2}\left[S_{+} S_{3}^{N-2}+S_{3} S_{+} S_{3}^{N-3}+\ldots S_{3}^{N-2} S_{+}\right]
$$

where the $c_{i}(r)$ are complex numbers, so that

$$
C^{(N) \dagger}=c_{0}^{*} S_{-}+c_{1}^{*}\left[S_{-} S_{3}+S_{3} S_{-}\right]+\ldots+c_{N-2}^{*}\left[S_{-} S_{3}^{N-2}+\ldots S_{3}^{N-2} S_{-}\right] .
$$

The next stage is to rewrite this ansatz in a form suitable for taking the limit $N \rightarrow \infty$. Following [7] we now write the matrices $A, B, C$ and $D$ in terms of the matrix polynomials

$$
\tilde{T}_{l, m}^{(N)}=\sum_{i_{k}=1,2,3} a_{i_{1} \ldots i_{l}}^{(m)} S_{i_{1}} \ldots S_{i_{l}}
$$


for $l=1, \ldots N-1, m=-l, \ldots l$, where $a_{i_{1} \ldots i_{l}}^{(m)}$ is a symmetric and traceless tensor given by the spherical harmonics:

$$
Y_{l, m}(\theta, \phi)=\sum_{\substack{i_{k}=1,2,3 \\ k=1, \ldots l}} a_{i_{1} \ldots i_{l}}^{(m)} \eta_{i_{1}} \ldots \eta_{i_{l}}
$$

where $\eta_{1}=\cos \phi \sin \theta, \eta_{2}=\sin \phi \sin \theta, \eta_{3}=\cos \theta$. Using the variables $\eta_{+}=e^{i \phi} \sin \theta=$ $\eta_{1}+i \eta_{2}, \eta_{-}=e^{-i \phi} \sin \theta=\eta_{1}-i \eta_{2}$, the relevant spherical harmonics are:

$$
\begin{aligned}
Y_{l, 0}(\theta, \phi) & =\left[\frac{2 l+1}{4 \pi}\right]^{\frac{1}{2}} P_{l}(\cos \theta) \\
Y_{l, 1}(\theta, \phi) & =-\left[\frac{2 l+1}{4 \pi} \frac{(l-1) !}{(l+1) !}\right]^{\frac{1}{2}} P_{l}^{1}(\cos \theta) e^{i \phi} \\
Y_{l,-1}(\theta, \phi) & =-\left[\frac{2 l+1}{4 \pi} \frac{(l-1) !}{(l+1) !}\right]^{\frac{1}{2}} P_{l}^{1}(\cos \theta) e^{-i \phi} ;
\end{aligned}
$$

where

$$
P_{l}(\cos \theta)=\frac{1}{2^{l} l !}\left(\frac{d}{d(\cos \theta)}\right)^{l}\left(\cos ^{2} \theta-1\right)^{l}
$$

is a Legendre polynomial, and

$$
P_{l}^{1}(\cos \theta)=\left(1-\cos ^{2} \theta\right)^{\frac{1}{2}}\left(\frac{d}{d(\cos \theta)}\right) P_{l}(\cos \theta)
$$

is an associated Legendre function. Since $P_{l}(\cos \theta)$ is a polynomial of order $l$, then $P_{l}^{1}=$ $\sin \theta \times$ a polynomial of order $l-1$, so that

$$
\begin{aligned}
Y_{l, 0}(\theta, \phi) & \propto P_{l}\left(\eta_{3}\right) \\
Y_{l, 1}(\theta, \phi) & \propto \eta_{+} P_{l}^{\prime}\left(\eta_{3}\right) \\
Y_{l,-1}(\theta, \phi) & \propto \eta_{-} P_{l}^{\prime}\left(\eta_{3}\right) .
\end{aligned}
$$

The spherical harmonics in this form are not homogeneous (as required by (13)), however they can be made homogeneous by including suitable powers of $\eta_{1}^{2}+\eta_{2}^{2}+\eta_{3}^{2}=1$.

The ansatz is then

$$
\begin{aligned}
A^{(N)} & =\tilde{a}_{0}^{N} \tilde{T}_{0,0}^{(N)}+\tilde{a}_{1}^{N} \tilde{T}_{1,0}^{(N)}+\ldots+\tilde{a}_{N-1}^{N} \tilde{T}_{N-1,0}^{(N)} \\
B^{(N)} & =\tilde{b}_{0}^{N} \tilde{T}_{0,0}^{(N)}+\tilde{b}_{1}^{N} \tilde{T}_{1,0}^{(N)}+\ldots+\tilde{b}_{N-1}^{N} \tilde{T}_{N-1,0}^{(N)} \\
C^{(N)} & =\tilde{c}_{1}^{N} \tilde{T}_{1,1}^{(N)}+\tilde{c}_{2}^{N} \tilde{T}_{2,1}^{(N)}+\ldots+\tilde{c}_{N-1}^{N} \tilde{T}_{N-1,1}^{(N)} \\
D^{(N)} & =\tilde{d}_{1} \tilde{T}_{1,0}^{(N)} .
\end{aligned}
$$


As before, $\tilde{a}_{i}, \tilde{b}_{i}$ are purely imaginary, $\tilde{c}_{i}$ are complex, and $\tilde{d}_{1}$ is real. Due to the selfadjointness of $S_{i}, i=1,2,3$. the $\tilde{T}$ satisfy $\left[\tilde{T}_{l, m}^{(N)}\right]^{\dagger}=(-1)^{m} \tilde{T}_{l,-m}^{(N)}$. Hence,

$$
C^{(N) \dagger}=-\left(\tilde{c}_{1}^{(N)}\right)^{*} \tilde{T}_{1,-1}^{(N)}-\ldots-\left(\tilde{c}_{N-1}^{(N)}\right)^{*} \tilde{T}_{N-1,-1}^{(N)} .
$$

In order for the theory to have a well-defined limit as $N \rightarrow \infty$, one should form a new basis [7, 14] for the $S U(N)$ group, $\left\{T_{l, m}^{(N)}\right\}$, by replacing $S_{i}$ in $\tilde{T}_{l, m}^{(N)}$ by $\tau_{i}$ defined by:

$$
\tau_{i} \equiv \frac{1}{N} S_{i}
$$

so that the ansatz (18) now has the form

$$
A^{(N)}=a_{0}^{(N)} T_{0,0}^{(N)}+a_{1} T_{1,0}^{(N)}+\ldots a_{N-1}^{(N)} T_{N-1,0}^{(N)}
$$

and similarly for the other matrices. Note that $A^{(N)}$ as defined here corresponds to a rescaled gauge potential, as compared with that in ref. [13], by a factor $\frac{1}{N}$; this is due to the fact that $A^{(N)}$ has a finite limit as $N \rightarrow \infty$. We are now in a position simply to write down the ansatz for the $S U(\infty)$ gauge field. The result is:

$$
A=\sum_{l=1}^{\infty} a_{l} Y_{l, 0} \quad B=\sum_{l=1}^{\infty} b_{l} Y_{l, 0} \quad C=\sum_{l=1}^{\infty} c_{l} Y_{l, 1} \quad D=d_{1} Y_{1,0},
$$

where $a_{l}, b_{l}$ and $c_{l}$ depend on $r$, and $d_{1}$ is a real constant.

\subsection{Field Equations for Einstein-SU( $\infty)$ Yang-Mills Theory}

The Einstein and Yang-Mills equations for an $S U(N)$ gauge theory, in a sphericallysymmetric space-time, have been derived in ref. [13]. It is the point of this subsection to re-derive these equations in the limit $N \rightarrow \infty$. To this end we first start by briefly reviewing the results of ref. [13], that are relevant for our purposes here. For more details on the finite- $N S U(N)$ gauge theory we refer the interested reader to ref. [13].

Firstly, we consider the Yang-Mills equations. One component of these leads to the following equation, for the ansatz (8), where none of the $\omega_{j}^{(N)}$ vanish,

$$
\mathcal{B}_{j}^{(N)}+\gamma_{j}^{\prime(N)}=0 .
$$

So far we have not fixed our choice of gauge, so we use the remaining gauge freedom we have to set $\mathcal{B}_{j}^{(N)}=0$ for static solutions [15], and then on account of (23) one has $\gamma_{j}^{\prime(N)}=0$. This implies that each $\gamma_{j}^{(N)}$ is a constant, which we set equal to 0 for simplicity. In addition, we shall concentrate on purely magnetic field configurations by setting $\mathcal{A}_{j}^{(N)}=0$ for all $j$. 
In order to obtain a finite limit of the field equations as $N \rightarrow \infty$, care is needed with factors of $N$. The ansatz used in the equations below is (21), with the basis of $S U(N)$ matrices constructed from the rescaled matrices $\tau_{i}(20)$. The field equations can then be taken from [13, making the following substitution [7]

$$
\hat{\mathcal{A}} \rightarrow N \hat{\mathcal{A}}
$$

We consider a spherically symmetric geometry described by the following metric, in Schwarzschild co-ordinates:

$$
d s^{2}=-\mu e^{2 \delta(r)} d t^{2}+\mu^{-1} d r^{2}+r^{2}\left(d \vartheta^{2}+\sin ^{2} \vartheta d \varphi^{2}\right)
$$

where the metric function $\mu$ has the form

$$
\mu=1-\frac{2 m(r)}{r}-\frac{\Lambda r^{2}}{3}
$$

and we have introduced a cosmological constant $\Lambda$. It should be emphasised at this point that the co-ordinates $\vartheta, \varphi$ are different from the "internal" variables $\theta, \phi$, which appeared in the ansatz for the $S U(\infty)$ gauge fields in the previous section. For finite $N$, the remaining Yang-Mills equation is, with ' denoting derivative with respect to $r$,

$$
r^{2} \mu C^{\prime \prime}+\left(2 m-\frac{2 \Lambda r^{3}}{3}-\kappa r^{3} p_{\vartheta}\right) C^{\prime}+C+\frac{1}{2} N^{2}\left[C,\left[C, C^{\dagger}\right]\right]=0
$$

where $\kappa$ is the gravitational coupling constant (we have set the gauge field coupling constant to unity), and

$$
p_{\vartheta}=\frac{1}{r^{4}} \operatorname{Tr}\left(D-N\left[C, C^{\dagger}\right]\right)^{2} .
$$

The Einstein equations read

$$
m^{\prime}=\frac{\kappa}{2}\left(\mu G+r^{2} p_{\vartheta}\right), \quad \delta^{\prime}=\frac{\kappa}{r} G
$$

where

$$
G=\operatorname{Tr}\left(C^{\prime} C^{\prime \dagger}\right)
$$

The presence of the explicit factors of $N$ is due to the substitution (24) and will be essential for obtaining the limit $N \rightarrow \infty$.

In taking the limit $N \rightarrow \infty$, the matrix $C$ becomes a function of the internal coordinates $\theta, \phi$ (i.e. those variables which are the arguments of the spherical harmonics, 
rather than the space-time co-ordinates $\vartheta$ and $\varphi$ ). The following substitutions are made [D]:

$$
\begin{aligned}
\operatorname{Tr} & \rightarrow \frac{1}{4 \pi} \int_{\theta=0}^{\pi} \int_{\phi=0}^{2 \pi} \sin \theta d \theta d \phi \\
N[P, Q] & \rightarrow i\{P(r, \theta, \phi), Q(r, \theta, \phi)\},
\end{aligned}
$$

where the Poisson bracket is defined as:

$$
\{P, Q\}=\frac{\partial P}{\partial(\cos \theta)} \frac{\partial Q}{\partial \phi}-\frac{\partial P}{\partial \phi} \frac{\partial Q}{\partial(\cos \theta)} .
$$

The Yang-Mills equation (27) therefore takes the form

$$
r^{2} \mu \frac{\partial^{2} C}{\partial r^{2}}+\left(2 m-\frac{2 \Lambda r^{3}}{3}-\kappa r^{3} p_{\vartheta}\right) \frac{\partial C}{\partial r}+C-\frac{1}{2}\left\{C,\left\{C, C^{\dagger}\right\}\right\}=0
$$

while the Einstein equations retain the form (29), with

$$
\begin{aligned}
G & =\frac{1}{4 \pi} \int_{\theta=0}^{\pi} \int_{\phi=0}^{2 \pi} \frac{\partial C}{\partial r} \frac{\partial C^{\dagger}}{\partial r} \sin \theta d \theta d \phi \\
p_{\vartheta} & =\frac{1}{16 \pi r^{4}} \int_{\theta=0}^{\pi} \int_{\phi=0}^{2 \pi}\left[D-i\left\{C, C^{\dagger}\right\}\right]^{2} \sin \theta d \theta d \phi .
\end{aligned}
$$

The ansatz of the previous section (22) can be written as:

$$
C(r, \theta, \phi)=f(r, \theta) e^{i \phi}, \quad D=d_{1} \cos \theta
$$

where $f$ is a real function and $d_{1}$ a real constant. The generalization of the Künzle 13 ansatz for $S U(N)$ (6) would imply that $d_{1}=2$. However, in order for $D$ to satisfy the $S U(\infty)$ equivalent of the conditions (5), namely,

$$
i\{D, C\}=2 C, \quad i\left\{D, C^{\dagger}\right\}=-2 C^{\dagger}
$$

it must be the case that $d_{1}=-2$. We shall take this value of $d_{1}$ when we consider solutions of the field equations in the next section.

In terms of the co-ordinate $\xi=\cos \theta$, the Yang-Mills equation can be written as:

$$
r^{2} \mu \frac{\partial^{2} f}{\partial r^{2}}+\left(2 m-\frac{2 \Lambda r^{3}}{3}-\kappa r^{3} p_{\vartheta}\right) \frac{\partial f}{\partial r}+f+\frac{1}{2} f \frac{\partial^{2}}{\partial \xi^{2}}\left(f^{2}\right)=0 ;
$$


and the quantities (34) simplify to:

$$
\begin{aligned}
G & =\frac{1}{2} \int_{\xi=-1}^{1}\left(\frac{\partial f}{\partial r}\right)^{2} d \xi \\
p_{\vartheta} & =\frac{1}{8 r^{4}} \int_{\xi=-1}^{1}\left(d_{1} \xi-\frac{\partial}{\partial \xi}\left(f^{2}\right)\right)^{2} d \xi
\end{aligned}
$$

This is the simplest form of the equations for our purposes. The function $f$ is further restricted by the ansatz (22) to be of the form

$$
f(r, \xi)=\sqrt{1-\xi^{2}} \sum_{i=1}^{\infty} f_{i}(r) \xi^{i} .
$$

In the next section we shall consider black hole solutions of the equations (29,37,38).

\section{Black hole solutions of the $S U(\infty)$ field equations}

We now turn to the solutions of the field equations (29,37,38), focusing our attention on black hole geometries. These equations are rather complicated, since they involve a

non-linear partial differential equation and integro-differential equations. Given the fact that the $S U(N)$ equations have only been solved numerically, tackling the field equations we have here would require a considerable amount of complex numerical calculations. The approach we shall take here is firstly to consider the special cases, namely the embedded Schwarzschild, Reissner-Nordström and $S U(2)$ black holes, in order to check our formalism and fix the remaining constants. Then we shall employ an asymptotic expansion in $\epsilon=1 / \Lambda$ for the case $\Lambda<0,|\Lambda| \rightarrow \infty$. This technique will enable us to find analytic, perturbative solutions which, it is hoped, will have many of the physical properties of the more general exact solutions but will be more amenable to analysis. We are looking for black hole solutions with a regular event horizon at $r=r_{h}$, and we want the geometry to approach (the covering space of) anti-de Sitter space (AdS) as $r \rightarrow \infty$. In the final subsection we shall make the alternative approximation of a very small gauge field $(|f| \ll$ 1 ), in which case the field equations again simplify considerably, for all negative values of $\Lambda$. This situation is particularly relevant in view of holography and the AdS/CFT correspondence 10, 11].

\subsection{Embedded $S U(2)$ solutions}

Before discussing the genuine $S U(\infty)$ solutions of the field equations $(29,37,38)$, we first consider embedded $S U(2)$ solutions. For all $N$, it is known that $S U(N)$ EYM theory 
has neutral embedded $S U(2)$ solutions as well as charged embedded $S U(k)$ solutions for $k=1, \ldots, N-1[8]$. The neutral $S U(2)$ solutions can be readily embedded into $S U(\infty)$ EYM. However, this is not possible for the charged solutions, whatever the value of $k$, including $k=2$.

The Yang-Mills function $f(r, \xi)$ is taken to be

$$
f(r, \xi)=w(r) \sqrt{1-\xi^{2}} .
$$

Then the Yang-Mills equation (37) reduces to:

$$
r^{2} \mu w^{\prime \prime}+\left(2 m-\frac{2 \Lambda r^{3}}{3}-\kappa r^{3} p_{\vartheta}\right) w^{\prime}+w\left(1-w^{2}\right)=0,
$$

and the quantities in the Einstein equations are:

$$
G=\frac{2}{3} w^{\prime 2}, \quad p_{\vartheta}=\frac{1}{3 r^{4}}\left(1-w^{2}\right)^{2},
$$

where we have fixed $d=-2$ as discussed in the previous subsection. These equations are exactly the same as those for $S U(2) \mathrm{EYM}$ if we set $\kappa=3$. This value of $\kappa$ is surprising, since it is conventional in studies of $S U(N)$ EYM (see, for example [8]) to take $\kappa=2$. The factor of 3 arises from integrating the $\sqrt{1-\xi^{2}}$ term, which does not arise in $S U(2)$ EYM. In the following we shall take $\kappa=3$ in order that we can check our results by comparison with the $S U(2)$ case.

There are two further special cases of the $S U(2)$ embedded solutions which should be mentioned. Firstly, setting $\omega \equiv \pm 1$ gives the Schwarzschild-AdS geometry, whilst setting $\omega \equiv 0$ gives a non-extremal Reissner-Nordström-AdS black hole with charge $Q=1$.

\subsection{Solutions for $|\Lambda| \gg 1$}

We now consider genuine $S U(\infty)$ solutions of the field equations (29, 37,38). In order to generate analytic solutions, we shall consider the case where $\Lambda<0,|\Lambda| \gg 1$. For $S U(2)$ EYM theory, the solutions are particularly simple in this limit, and have attractive properties [12]. In particular, there are solutions which are linearly stable. This limit corresponds to large black holes, which possess a stable Hartle-Hawking state 16]. Therefore, these are the objects we are particularly interested in if one wishes to consider issues of information loss during the evaporation process. Also, in this limit stringy corrections in the bulk geometry of AdS are negligible [10, 11], and the field theory limit is sufficient. 
Firstly, define a new parameter $\epsilon$ and a new variable $q$ by

$$
\epsilon=\frac{1}{\Lambda}, \quad q(r)=\frac{m(r)}{\Lambda},
$$

where the variable $q$, although it is a metric function, will be negative because $\Lambda<0$. Then the field equations (29,37,38) take the form

$$
\begin{aligned}
q^{\prime}(r) & =\frac{3}{2}\left(\epsilon-\frac{2 q}{r}-\frac{r^{2}}{3}\right) G+\frac{3}{2} r^{2} \epsilon p_{\vartheta} \\
\delta^{\prime}(r) & =\frac{3 G}{r} \\
0 & =r^{2}\left(\epsilon-\frac{2 q}{r}-\frac{r^{2}}{3}\right) \frac{\partial^{2} f}{\partial r^{2}}+\left(2 q-\frac{2 r^{3}}{3}-3 \epsilon r^{3} p_{\vartheta}\right) \frac{\partial f}{\partial r}+\epsilon f+\frac{1}{2} \epsilon f \frac{\partial^{2}}{\partial \xi^{2}}\left(f^{2}\right),
\end{aligned}
$$

where we have set the gravitational coupling constant $\kappa=3$ in order to obtain the correct equations for the $S U(2)$ embedding, as discussed in the previous subsection. We shall consider solutions for $|\epsilon| \ll 1$, and perform an asymptotic expansion for small $\epsilon$ as follows:

$$
\begin{aligned}
q(r) & =-\frac{r_{h}^{3}}{6}+\epsilon q_{1}(r)+\epsilon^{2} q_{2}(r)+\ldots \\
\delta(r) & =1+\epsilon \delta_{1}(r)+\epsilon^{2} \delta_{2}(r)+\ldots \\
f(r, \xi) & =f_{0}(\xi)+\epsilon f_{1}(r, \xi)+\epsilon^{2} f_{2}(r, \xi) .+\ldots
\end{aligned}
$$

When $\epsilon=0$, the solution is a Schwarzschild-AdS black hole with horizon radius $r_{h}$, and the gauge field $f$ is an arbitrary function of $\xi$ of the form (39). Here we are using an asymptotic expansion, in other words the first few terms of the expansion above should be an increasingly good approximation to the exact solutions as $\epsilon \rightarrow 0$, and the number of terms of the expansion which are useful will also increase as $\epsilon$ decreases. This approximation will be reliable as long as the terms in the expansions remain uniformly bounded in $r$. This is a strong assumption, but one which is valid for the terms in the expansion we obtain here. The advantage of the expansion is that each term in the metric functions $q$ and $\delta$ depends only on the previous terms in the expansion of the gauge function $f$, and for each term in $f$, we have a differential equation involving only derivatives with respect to $r$ for that term. Therefore it is comparatively straightforward to find solutions in the form (45) to whatever order in $\epsilon$ we like. We shall find that interesting effects occur if we work to second order in the metric functions and first order in the gauge field function.

To first order in $\epsilon$, we have:

$$
\delta_{1}^{\prime}(r)=0
$$




$$
\begin{aligned}
q_{1}^{\prime}(r) & =\frac{3 C_{1}}{4 r^{2}} \\
\mathcal{F}_{1}(\xi) & =r^{2}\left(r^{2}-\frac{r_{h}^{3}}{r}\right) \frac{\partial^{2} f_{1}}{\partial r^{2}}+\left(r_{h}^{3}+2 r^{3}\right) \frac{\partial f_{1}}{\partial r}
\end{aligned}
$$

where $C_{1}$ is a constant, and $\mathcal{F}_{1}$ a function of $\xi$, given by

$$
C_{1}=\int_{\xi=-1}^{1}\left[\xi+\frac{1}{2} \frac{\partial}{\partial \xi}\left(f_{0}^{2}\right)\right]^{2} d \xi, \quad \mathcal{F}_{1}=3\left[f_{0}+\frac{1}{2} f_{0} \frac{\partial^{2}}{\partial \xi^{2}}\left(f_{0}^{2}\right)\right] .
$$

The first two equations in (46) can be integrated straight away to give

$$
\delta_{1}(r)=0, \quad q_{1}(r)=K_{1}-\frac{3 C_{1}}{4 r}
$$

where $K_{1}$ is an arbitrary constant and we have fixed the constant arising in $\delta_{1}$ to be zero so that the geometry has the correct asymptotic form at infinity. The constant $K_{1}$ will be fixed by the requirement that there is a regular event horizon at $r=r_{h}$. This means that $q_{1}=\frac{r_{h}}{2}, q_{i}=0$ for $i \geq 2$ at $r_{h}$. The form (48) corresponds to a Reissner-NordströmAdS geometry, as would have been expected. We will therefore need to go to the next order in the metric functions to get solutions with hair. Integrating the equation for the Yang-Mills field in (46) gives

$$
\frac{\partial f_{1}}{\partial r}=\frac{1}{r^{3}-r_{h}^{3}}\left(\mathcal{C}(\xi) r-\mathcal{F}_{1}(\xi)\right)
$$

where $\mathcal{C}$ is an arbitrary function. We require $f_{1}$ and all its derivatives to be regular everywhere outside and on the event horizon, which fixes $\mathcal{C}$ as:

$$
\mathcal{C} r_{h}=\mathcal{F}_{1}
$$

Integrating again then yields

$$
f_{1}(r, \xi)=\mathcal{F}_{2}(\xi)+\frac{2 \mathcal{F}_{1}}{r_{h}^{2} \sqrt{3}} \tan ^{-1}\left(\frac{2 r+r_{h}}{r_{h} \sqrt{3}}\right),
$$

with another arbitrary function $\mathcal{F}_{2}(\xi)$. Note that from $(47), \mathcal{F}_{1}$ is of the form (39), so if we choose $\mathcal{F}_{2}$ to also be written as (39), then the whole gauge field will have the structure required. The function $f_{1}$ above consists of genuine $S U(\infty)$ gauge field hair. It contains two arbitrary functions of $\xi$ (subject to the restrictions just discussed), which require an infinite number of constants in order to specify them (which correspond to the coefficients in the expansion (39)). 
We now want to find the effect on the geometry of the gauge field hair (51). To second order, the Einstein equations give the following equations for the metric perturbations:

$$
\begin{aligned}
\delta_{2}^{\prime}(r) & =\frac{3 C_{2}}{2 r r_{h}^{2}}\left(r^{2}+r r_{h}+r_{h}^{2}\right)^{-2} \\
q_{2}^{\prime}(r) & =\frac{C_{2}}{4 r r_{h}^{2}} \frac{r_{h}-r}{r^{2}+r r_{h}+r_{h}^{2}}+\frac{3 C_{3}}{r^{2}}+\frac{2 \sqrt{3} C_{4}}{r^{2} r_{h}^{2}} \tan ^{-1}\left(\frac{2 r+r_{h}}{r_{h} \sqrt{3}}\right)
\end{aligned}
$$

where we have defined new constants $C_{2}-C_{4}$ by

$$
\begin{aligned}
C_{2} & =\int_{\xi=-1}^{1} \mathcal{F}_{1}^{2}(\xi) d \xi \\
C_{3} & =\int_{\xi=-1}^{1}\left(\xi+\frac{1}{2} \frac{\partial}{\partial \xi}\left(f_{0}^{2}\right)\right) \frac{\partial}{\partial \xi}\left(f_{0} \mathcal{F}_{2}\right) d \xi \\
C_{4} & =\int_{\xi=-1}^{1}\left(\xi+\frac{1}{2} \frac{\partial}{\partial \xi}\left(f_{0}^{2}\right)\right) \frac{\partial}{\partial \xi}\left(f_{0} \mathcal{F}_{1}\right) d \xi
\end{aligned}
$$

Integrating then gives

$$
\begin{aligned}
\delta_{2}(r)= & \frac{3 C_{2}}{4 r_{h}^{6}}\left[\log \left(\frac{r^{2}}{r^{2}+r r_{h}+r_{h}^{2}}\right)+\frac{2 r_{h}\left(r_{h}-r\right)}{3\left(r^{2}+r r_{h}+r_{h}^{2}\right)}-\frac{10}{3 \sqrt{3}} \tan ^{-1}\left(\frac{2 r+r_{h}}{r_{h} \sqrt{3}}\right)\right]+K_{2} \\
q_{2}(r)= & \frac{1}{8 r_{h}^{3}}\left(C_{2}+12 C_{4}\right) \log \left(\frac{r^{2}}{r^{2}+r r_{h}+r_{h}^{2}}\right)-\frac{3 C_{3}}{r} \\
& -\frac{1}{4 r_{h}^{3}}\left(\sqrt{3} C_{2}+12 C_{4}+\frac{24 C_{4} r_{h}}{r}\right) \tan ^{-1}\left(\frac{2 r+r_{h}}{r_{h} \sqrt{3}}\right)+K_{3}
\end{aligned}
$$

where $K_{2}$ and $K_{3}$ are arbitrary constants. The constant $K_{2}$ is fixed by the requirement that $\delta_{2} \rightarrow 0$ as $r \rightarrow \infty$ to be

$$
K_{2}=\frac{5 \pi C_{2}}{4 \sqrt{3} r_{h}^{6}},
$$

whilst the constant $K_{3}$ will be fixed by the requirement that $q_{2}=0$ at $r=r_{h}$ so that we have a regular event horizon. We now have a hairy black hole metric, caused by the coupling of the geometry to the $S U(\infty)$ Yang-Mills field. In figure 1 we compare the approximate solution (51), with the exact numerical solution in the $S U(2)$ case, where $f$ has the form (40). We use parameters $\Lambda=-100$ and $w\left(r_{h}\right)=0.8$. It can be seen that the approximate solution converges a little too quickly, but otherwise has the same qualitative features as the exact solution in this case. The approximation would become better if we used more terms in the expansion (45), or if $|\Lambda|$ was larger.

At this stage, a comment is in order concerning the nature of the hair we have found. In particular, we wish to stress that the hair is definitely infinite in quantity, and that 
an infinite number of parameters is required in order to describe it. As mentioned above, the gauge field requires an infinite number of constants for it to be specified in this approximation, since two arbitrary functions of $\xi$ are involved. Each subsequent term in the expansion in $\epsilon$ will introduce a further function of $\xi$ into the gauge field, and hence a further infinite number of quantities. Suppose, however, that we wish to fix the behaviour of the gauge field at, say, the event horizon (this was the procedure used to fix the constants in order to obtain figure 1, and is more convenient computationally than fixing quantities at infinity). This would require an infinite number of quantities to specify, say $f_{0}(\xi)$. The subsequent functions of $\xi$ would be fixed by requiring that $f_{i}(r, \xi)=0$ at $r=r_{h}$ for $i>0$, for example this would mean that

$$
\mathcal{F}_{2}(\xi)=\frac{2 \pi \sqrt{3} \mathcal{F}_{1}(\xi)}{r_{h}^{2}} .
$$

The result, either way, is that an infinite number of parameters are needed to specify the gauge field function. Next we turn to the metric functions. These do not depend on $\xi$, but do contain constants constructed from the gauge field function by integrating over $\xi$. Each term in the perturbation expansion of the metric functions involves at least one new constant constructed from the gauge field function (which cannot be determined from previous constants) as well as a constant of integration. The constants of integration can easily be fixed by the boundary conditions on the metric i.e. by setting $\delta_{i} \rightarrow 0$ as $r \rightarrow \infty$ for $i>0$ so that the geometry has the correct behaviour at infinity, and setting $q_{i}=0$ at $r=r_{h}$ for $i \geq 2$ so that there is a regular event horizon at $r=r_{h}$. However, an infinite number of different constants, coming from the gauge field function, will be required if we are to specify the metric to all orders in $\epsilon$. Thus, while it appears that we only need a finite number of constants to construct the metric to each order in perturbation theory, the exact metric will need an infinite number of constants, so that we do have infinite amounts of hair.

\subsection{Solutions with a small gauge field}

In the previous subsection, we performed an asymptotic expansion for very large $|\Lambda|$, and found analytic solutions representing black holes dressed with infinite amounts of gauge field hair. We now turn to another situation in which we can find solutions easily, and which is of direct relevance to holography and the AdS/CFT correspondence [10, 11] (which partly motivates our consideration of black holes in AdS). The holographic principle (namely that dynamics of gauge fields in the bulk of AdS is determined by what is happening on the boundary) relies on a theorem of Graham and Lee [17] which applies only to gauge fields sufficiently close to zero [11]. We stated previously that if the gauge field $f \equiv 0$, then the geometry is that of non-extremal Reissner-Nordström-anti-de Sitter 
(RN AdS) space, with charge $Q=1$. Therefore in this section we are in effect considering perturbations about this configuration.

Let $\varepsilon$ be a small parameter such that $\varepsilon \ll 1$ and $|f(r, \xi)| \leq \varepsilon$ for all $r$, so that the magnitude of the gauge field is uniformly bounded and is close to zero. The parameter $\varepsilon$ determines the magnitude of the gauge field (no matter what the size of the cosmological constant $\Lambda$ ) and should not be confused with $\epsilon=1 / \Lambda$ of the previous section where $|\Lambda| \gg 1$. This condition of uniform boundedness is rather strong, as we shall see shortly, and the negative cosmological constant is crucial if this condition is to be met. We then consider the following asymptotic expansions of the field variables as $\varepsilon \rightarrow 0$ :

$$
\begin{aligned}
f(r, \xi) & =\varepsilon\left[f_{0}(r, \xi)+\varepsilon f_{1}(r, \xi)+\varepsilon^{2} f_{2}(r, \xi)+\ldots\right] \\
m(r) & =m_{0}(r)+\varepsilon m_{1}(r)+\varepsilon^{2} m_{2}(r)+\ldots \\
\delta(r) & =\varepsilon \delta_{1}(r)+\varepsilon^{2} \delta_{2}(r)+\ldots
\end{aligned}
$$

These expansions are inserted into the field equations (29, 37, 38), as a consequence of which $f_{1}, m_{1}$ and $\delta_{1}$ all vanish identically. In addition, we have, to lowest order, the RN AdS geometry, so that

$$
m_{0}(r)=\frac{1}{2 r_{h}}+\frac{r_{h}}{2}-\frac{\Lambda r_{h}^{3}}{6}-\frac{1}{2 r} .
$$

In fact this is a good approximation to the exact metric function $m$, as can be seen from figure 2, where we compare (58) with the exact numerical solution for the $S U(2)$ case with $\Lambda=-0.001$ and $w\left(r_{h}\right)=0.1$. Again, the approximate solution has the correct qualitative features and just converges a little too quickly.

The Yang-Mills equation, to lowest order, tells us that the $\xi$ dependence of $f_{0}$ is arbitrary (subject to (39)), but its $r$ dependence is governed by the following differential equation:

$$
r^{2}\left(1-\frac{2 m_{0}}{r}-\frac{\Lambda r^{2}}{3}\right) \frac{\partial^{2} f_{0}}{\partial r^{2}}+\left(2 m_{0}-\frac{2 \Lambda r^{3}}{3}-\frac{1}{r}\right) \frac{\partial f_{0}}{\partial r}+f_{0}=0 .
$$

This is exactly the same equation satisfied by very small $S U(2)$ gauge fields, and in this case we can see that the gauge field effectively becomes an infinite number of $S U(2)$ degrees of freedom. The interaction between the degrees of freedom does not come into play in this approximation because the gauge field is very small (and so we have ignored terms involving $f^{2}$ ).

We can now use our knowledge of $S U(2)$ EYM theory in AdS [12] to comment on what will happen in the $S U(\infty)$ case. From [12], there are solutions of $S U(2)$ EYM theory in 
which the gauge field is uniformly bounded and arbitrarily small. This is very different to the situation when $\Lambda=0$, when $w \rightarrow \pm 1$ as $r \rightarrow \infty$, so that any gauge field which is very small close to the event horizon, must become order unity at infinity. In this case our expansion in terms of $\varepsilon$ is not uniformly valid. For $\Lambda>0$, it is possible to have gauge fields which are uniformly arbitrarily small, however, in that case, the set of parameters $w\left(r_{h}\right)$ which lead to regular black hole solutions consists of discrete points [18]. When the cosmological constant is negative, we have regular black hole solutions for a continuous interval of values of $w\left(r_{h}\right)$ containing zero, although the size of this interval shrinks to zero as $\Lambda \rightarrow 0_{-}$[12]. Therefore, for any negative cosmological constant (no matter how small or large), we can specify any sufficiently small function $f(\xi)$ satisfying the ansatz (39) to be the gauge field function at the event horizon, and then we have infinite gauge field hair which remains arbitrarily small at all distances away from the black hole horizon, including up to the boundary of AdS. It is for this reason that we view the $\Lambda \rightarrow 0_{-}$ model as a "regulator" model for $S U(\infty)$-hair on asymptotically flat space-times with zero-cosmological constant. A zero-cosmological-constant situation might be desirable from both theoretical and phenomenological points of view, e.g. it may be the result of a space-time symmetry like supersymmetry. Notably, in the two-dimensional stringy analogue of the $W$-hair [4], the ground-state energy of the two-dimensional string has been preserved under the action of the $W_{\infty}$ symmetries [19] and this could be viewed as the analogue of a zero-cosmological constant situation.

\section{Conclusions}

In this work we have formulated the field equations for spherically-symmetric EYM black holes in $S U(\infty)$ gauge theory. Their study was motivated by some considerations in string theory, the holographic principle and the so-called AdS/CFT correspondence. We have solved these equations both numerically and analytically in some cases. The introduction of a negative cosmological constant (no matter how small) proved extremely useful in simplifying the equations by means of appropriate perturbative expansions. Specifically, we have obtained solutions for the cases: (i) $\Lambda<0$ with $|\Lambda| \gg 1$, and finite gauge fields, and (ii) $\Lambda<0$ arbitrary, and infinitesimally small gauge fields. The latter case exhibits holographic properties of the gauge-field hair and may be relevant for issues related to information loss.

However, as stressed in the introduction, the issue of quantum coherence is still far from being understood. The above holographic properties pertain to classical field configurations, and it is not clear that they survive a full quantum treatment, where space-time fluctuations are taken into account. In ref. 20] we have presented an approach to quan- 
tify entropy production during an evaporation process for the case of an $S U(2)$ EYM black hole, coupled to scalar matter [21]. This can be done by identifying the targetspace temporal evolution parameter, 'time' $t$, with the logarithm of the brick-wall scale, interpreted as a Renormalization-Group scale that regularizes ultraviolet (short-distance) infinities in the coupled matter/black hole system. One then concentrates on the logarithmic divergences in the expression for the entropy of the scalar field. Such divergences are extra divergences that cannot be absorbed in a standard renormalization of Newton's or other field-theory coupling constants (which for instance yield a regularized BekensteinHawking area law [21]). In that sense, such terms have been interpreted in [20] as entropy production.

If the holographic properties of the $S U(N \rightarrow \infty)$ EYM AdS background were to be extended to the quantum case, such logarithmic divergences should have been suppressed by inverse powers of $N \rightarrow \infty$. However, it can be easily seen that this is not the case for a minimally-coupled scalar field neutral under the gauge symmetry. Indeed, the geometries we consider are small perturbations near RN AdS black-hole space times, and therefore the entropy of a scalar field interacting with this geometry is very nearly the same as the RN AdS case, which is known to be non-trivial [22]. However, the case of a scalar field coupled to the gauge degrees of freedom, which exhibit the holographic properties, may completely change the picture. We hope to return to this issue in a future work. Nevertheless, we believe that the results presented in this work are of sufficient interest to motivate further studies in the above direction.

\section{Acknowledgements}

It is a pleasure to thank John Ellis for useful discussions and for suggesting the title ! The work of N.E.M. is partially supported by P.P.A.R.C. (U.K.). E.W. wishes to thank Oriel College (Oxford) for financial support and the Department of Physics, University of Newcastle (Newcastle-upon-Tyne) for hospitality. 


\section{References}

[1] R. Bartnik and J. McKinnon, Phys. Rev. Lett. 61 (1988) 141.

[2] P. Bizon, Phys. Rev. Lett. 64 (1990) 2844.

[3] J.H. Schwarz, Target Space Duality and the Curse of the Wormhole, Proc. Int. Conf. on High Energy Physics: Beyond the Standard Model-II, Norman, Oklahoma, November 1-3, 1990 (eds. K. A. Milton, R. Kantowski and M. A. Samuel, World Scientific, 1991) p. 384;

S. Kalara and D.V. Nanopoulos, Phys. Lett. B267 (1991) 343.

L. Susskind, Phys. Rev. D49 (1994) 6606; ibid. D52 (1995) 6997.

[4] J. Ellis, N.E. Mavromatos and D.V. Nanopoulos, Phys. Lett. B272 (1991) 261.

[5] I. Bakas, Phys. Lett. B228 (1989) 57; Comm. Math. Phys. 134 (1990) 487;

I. Bakas and E. Kiritsis, Int. J. Mod. Phys. A6 (1991) 2871, and references therein.

[6] L. Krauss and F. Wilczek, Phys. Rev. Lett. 62 (1986) 380;

S. Coleman, J. Preskill and F. Wilczek, Nucl. Phys. B378 (1992) 175.

[7] E.G. Floratos, J. Iliopoulos and G. Tiktopoulos, Phys. Lett. B217 (1989) 285.

[8] N.E. Mavromatos and E. Winstanley, J. Math. Phys. 39 (1998) 4849.

[9] G. 't Hooft, in Salamfest 1993, p. 284, gr-qc/9310026;

L. Susskind, J. Math. Phys. 36 (1995) 6377.

[10] J. Maldacena, Adv. Theor. Math. Phys. 2 (1998) 231.

[11] E. Witten, Adv. Theor. Math. Phys. 2 (1998) 253.

[12] E. Winstanley, Class. Quant. Grav. 16 (1999) 1963.

[13] H.P. Künzle, Class. Quant. Grav. 8 (1991) 2283.

[14] B. De Wit, J. Hoppe and H. Nicolai, Nucl. Phys. B305 (1988) 545.

[15] O. Brodbeck and N. Straumann, J. Math. Phys. 37 (1996) 1414.

[16] S.W. Hawking and D.N. Page, Comm. Math. Phys. 87 (1983) 577.

[17] R. Graham and J. Lee, Adv. Math. 87 (1991) 186. 
[18] M.S. Volkov, N. Straumann, G. Lavrelashvili, M. Heusler and O. Brodbeck, Phys. Rev. D54 (1996) 7243.

[19] E. Witten, Nucl. Phys. B373 (1992) 187.

[20] J. Ellis, N.E. Mavromatos, D.V. Nanopoulos and E. Winstanley, Mod. Phys. Lett. A12 (1997) 243.

[21] N.E. Mavromatos and E. Winstanley, Phys. Rev. D53 (1996) 3190.

[22] See, for instance: R.-G. Cai, Y.-Z. Zhang, Mod. Phys. Lett. A11 (1996) 2027. 


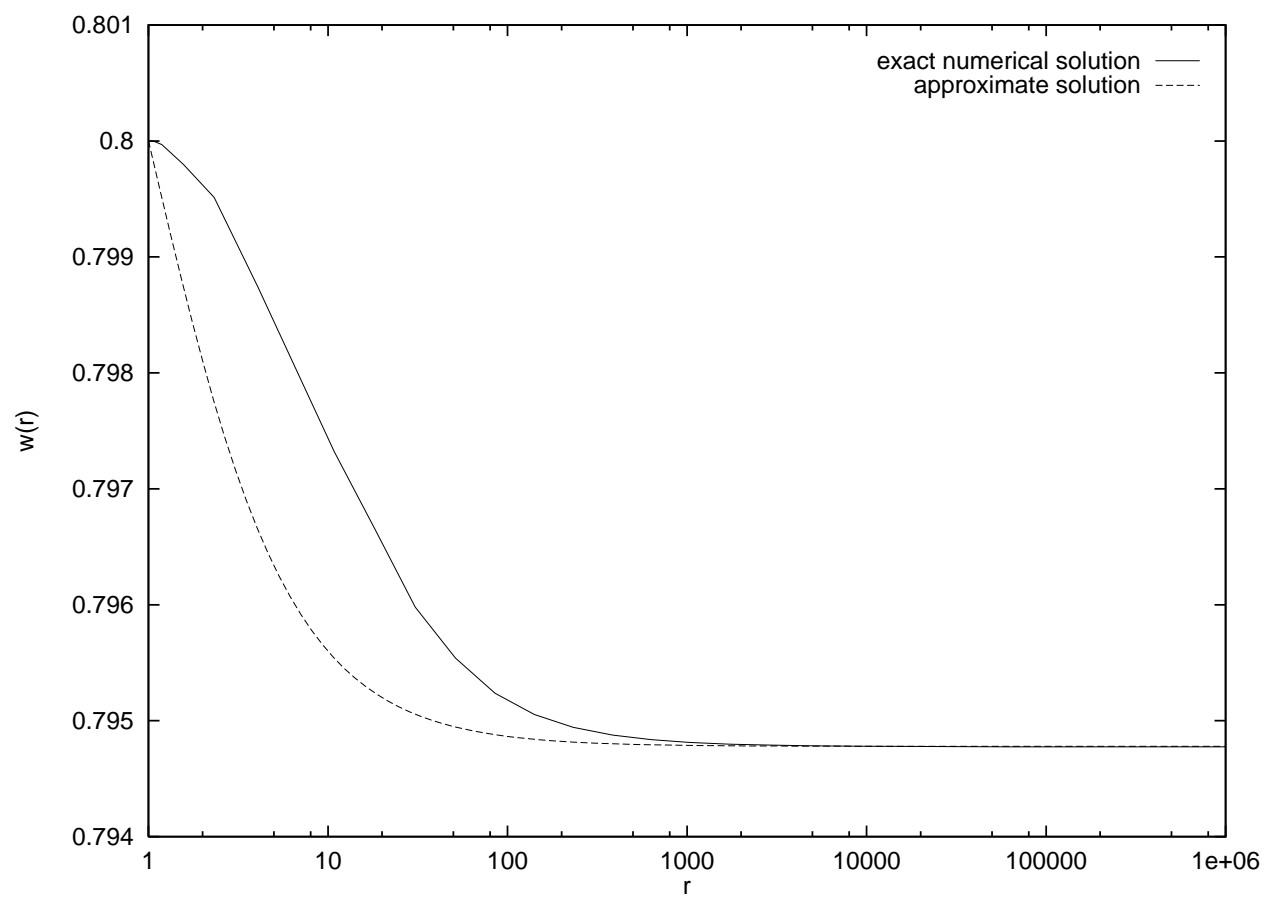

Figure 1: Comparison of the exact, numerical solution for the gauge field with gauge group $S U(2)$, where $\Lambda=-100$ and $w_{h}=0.8$ and the approximate solution given by (51). The approximate solution has the correct qualitative features, and simply converges a little too quickly. 


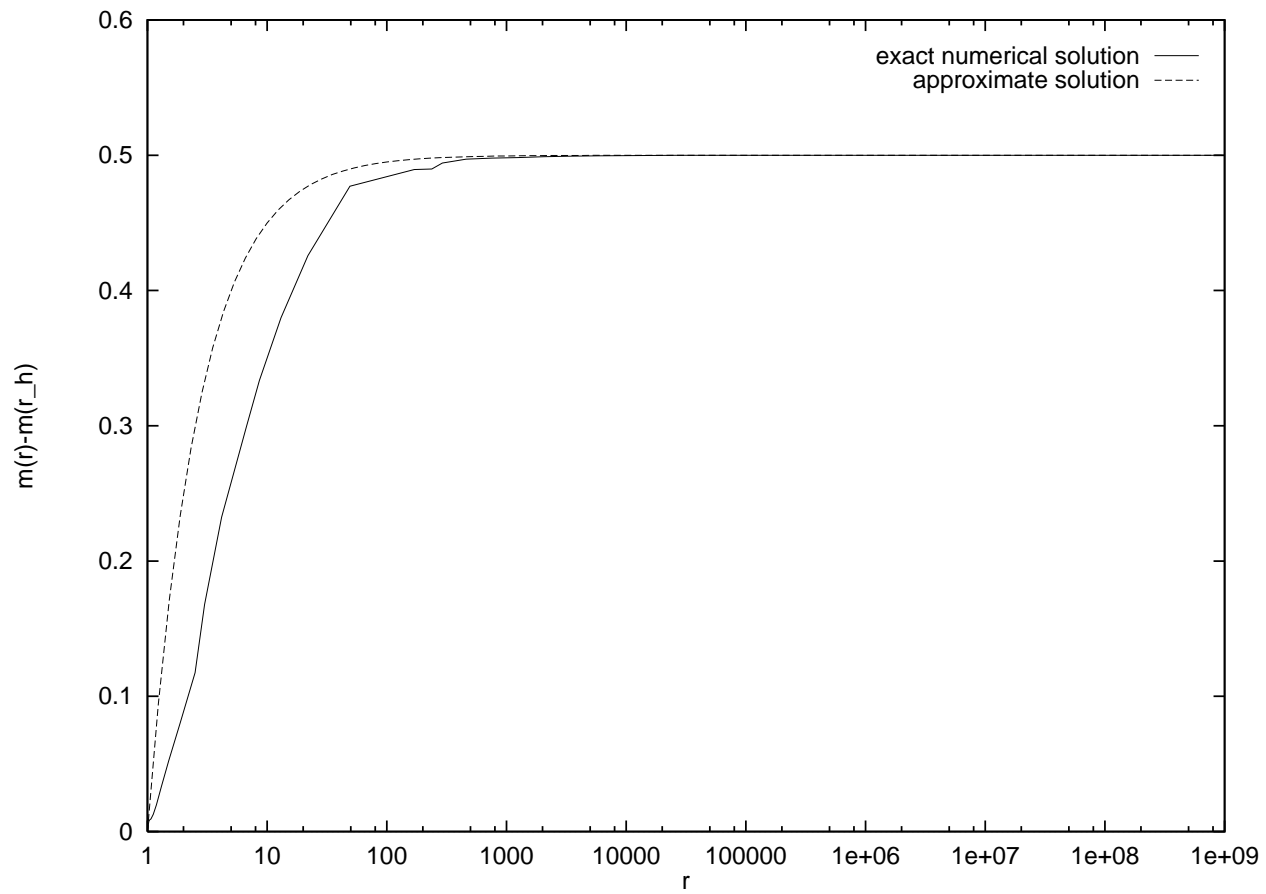

Figure 2: Comparison of the exact, numerical solution for the metric function $m(r)-m\left(r_{h}\right)$ with gauge group $S U(2)$, where $\Lambda=-0.001$ and $w\left(r_{h}\right)=0.1$ with the RN AdS solution, which is an approximation when the gauge field is small. From the figure it can be seen that the approximation is good in this case, even though the gauge field is not particularly small. 